\begin{tabular}{|l|l|l|l|l|l|}
\hline Intropica & ISSN 1794-161X & Vol. 13 & No.1 & $41-49$ & Santa Marta-Colombia, enero-junio de 2018 \\
\hline
\end{tabular}

\title{
MACROINVERTEBRADOS BENTÓNICOS COMO INDICADORES DE CALIDAD DE AGUA EN LA CUENCA DEL PACHANLICA, PROVINCIA DE TUNGURAHUA, ECUADOR
}

\author{
BENTHIC MACROINVERTEBRATES AS INDICATORS OF WATER QUALITY IN THE \\ PACHANLICA BASIN, TUNGURAHUA PROVINCE, ECUADOR
}

Marcia Buenaño (D), Carlos Vásquez, Hernán Zurita-Vásquez@iD, Giovana Parra@ y Ruth Pérez@D

\begin{abstract}
RESUMEN
Se determinó la abundancia de los macroinvertebrados bentónicos presentes en un río altoandino (microcuenca del Pachanlica), en la Provincia de Tungurahua, Ecuador, durante dos períodos de muestreo (abril-diciembre del 2010 y enero-junio del 2011). Durante cada período se realizaron seis muestreos y los especímenes recolectados fueron identificados hasta nivel de orden y familia. Fueron calculados dos índices: el índice BMWP (Biological Monitoring Working Party) y el índice EPT (Ephemeroptera, Plecoptera, Trichoptera) para conocer la calidad del agua en la microcuenca del Pachanlica. Se recolectó un total de 3023 especímenes de macroinvertebrados, de los cuales 1669 especímenes fueron recolectados durante abril-diciembre 2010 y 1354 en enero-junio 2011. Los valores del índice BMWP observados (variaron entre 2 y 34 y desde 0 y 36 ) durante el primer y segundo período, respectivamente que permiten considerar las aguas en condición muy crítica y crítica. Por otra parte, basados en los valores del índice EPT, los cuales variaron desde 0 a 4,82 (primer período) y desde 0 a 16,81 \% (segundo período), el agua de la cuenca Pachanlica podría categorizada como de mala calidad. La relativa poca abundancia de los macroinvertebrados podría tener relación con los parámetros físico-químicos del agua, principalmente con la concentración de amonio; sin embargo, se requieren estudios más detallados.
\end{abstract}

PALABRAS CLAVE: macroinvertebrados; parámetros físico-químicos; índices biológicos; Ecuador

\begin{abstract}
Abundance of the benthic macroinvertebrates occurring in a high Andean river (Pachanlica microbasin), Province of Tungurahua, Ecuador, was determined during two sampling periods (April-December 2010 and January-June 2011). Six samplings were carried out along each period and the collected specimens were identified up to order and family levels. Two indices were calculated: the BMWP index (Biological Monitoring Working Party) and the EPT index (Ephemeroptera, Plecoptera, Trichoptera) to figure out the water quality in the Pachanlica micro-basin. A total of 3023 macroinvertebrates were collected, being 1669 specimens collected during April-December 2010 and 1354 from January-June 2011. The observed BMWP index values (ranging from 2 to 34 or from 0 to 36) during the first and second periods, respectively, allowed to classify water quality as a very critical and critical condition. On the other hand, based on EPT index values which varied from 0 to $4.82 \%$ (first period) or 0 to $16.81 \%$ (second period), water in Pachanlica basin could be classified as poor quality. Relative low macroinvertebrates abundance could be related to the water's physic-chemical parameters, mainly to ammonia concentration; however more detailed study are required.
\end{abstract}

KEYS WORDS: macroinvertebrates; physic-chemical parameters; biological indexes; Ecuador

Dirección de los autores:

Universidad Técnica de Ambato. Facultad de Ciencias Agropecuarias. Campus Querochaca, Cantón Cevallos, Provincia de Tungurahua, Ecuador, e-mail: mbs.quim@hotmail.com (M.B.); e-mail: ca.vasquez@uta.edu.ec (C.V.); e-mail: jh.zurita@uta.edu.ec (H.Z.H). Universidad Técnica de Cotopaxi. Facultad de Ciencias Agropecuarias y Recursos Naturales. Campus Salache. Provincia de Latacunga, Ecuador, e-mail: giovana.parra@ utc.edu.ec (G.P.); e-mail: ruth.perez@utc.edu.ec (R.P). 


\section{INTRODUCCIÓN}

Las zonas altas andinas (1 000-3 500 msnm) constituyen actualmente una de las principales áreas de conservación debido a su alta riqueza biológica y alto nivel de endemismo, y adicionalmente porque es considerado uno de los ecosistemas menos conocidos de los trópicos (Armenteras et al., 2003). Sin embargo, se requieren la aplicación de prácticas de conservación debido a que sus cuencas hidrográficas están bajo presión extrema por las actividades de producción agrícola, y además las zonas en pendiente están expuestas al efecto de erosión severa del suelo (Monar et al., 2013).

El recurso de agua en los ecosistemas de alta montaña es muy sensible a cambios globales del clima y locales de uso del suelo (Buytaert et al., 2006). Así, las diversas actividades económicas provocan detrimento en la calidad del agua lo que reduce la disponibilidad de agua dulce, degrada el suelo, impacta numerosos ecosistemas terrestres y marinos y encarece el tratamiento de este vital elemento (Guimarães et al., 2012).

La fauna bentónica de los sistemas acuáticos continentales es una de las más ricas y probablemente entre las más diversificadas y juega un rol importante en el mantenimiento de la actividad metabólica de estos ambientes (Reinoso et al., 2008; Rivera et al., 2008; Montoya y Aguirre, 2009). Debido a que los macroinvertebrados bentónicos son sensibles a las variaciones ambientales, estos puedes ser usados para evaluar el grado de integridad ecológica del sistema tanto momentánea como estacional (Gutiérrez-Yurrita et al., 2002).

Las variaciones en las características naturales de los hábitats acuáticos determinan la composición de especies de plantas y animales acuáticos, los cuales pueden ser usados como bioindicadores de la calidad del ambiente (Seaonez, 1999). De acuerdo con Vázquez-Silva et al. (2006), los bioindicadores aportan información valiosa sobre la calidad del agua con menor costo en comparación con los métodos fisicoquímicos. Adicionalmente, el uso de macroinvertebrados resulta útil por su ubiquidad, condición sedentaria, lo que permite un análisis espacial del contaminante, ciclos de vida largos en comparación con otros grupos y son afectados por perturbaciones ambientales, lo que permite analizar cambios temporales causados por tales perturbaciones
Entre los grupos de organismos, los macroinvertebrados acuáticos han sido ampliamente usados en la evaluación de la influencia antrópica y en consecuencia permite determinar los niveles de calidad de cursos de agua (Norris y Hawkins, 2000). Tanto las poblaciones de peces como de macroinvertebrados encontrados en un ecosistema fluvial han desarrollado gran parte de su vida allí, asociándose a características típicas del agua, por lo que se constituyen en potenciales indicadores de su calidad (Jara, 2002). Estos organismos bentónicos se encuentran en todo tipo de ambiente acuático de agua dulce donde son importantes para el monitoreo de ese ecosistema acuático en particular (Cummnig y Klug, 1979).

A pesar de su destacada importancia, los ecosistemas dulceacuícolas han estado sometidos al impacto de la acción antropogénica, como el represamiento y remoción de la vegetación ribereña que ocasionan cambios drásticos en el flujo natural de la materia y la energía y modificaciones en el ciclo de nutrientes, especialmente del nitrógeno y fósforo y en la disponibilidad de sustratos orgánicos (Jorcin y Nogueira, 2008).

De acuerdo con Liñero-Arana et al. (2016), la baja calidad del agua en zonas altas del Ecuador posiblemente sea debida al uso de agroquímicos en los cultivos de ladera que, por efecto de lluvias, las aguas de escorrentía arrastrarían hasta los ríos. En consecuencia, es posible que las aguas servidas y residuos sólidos producto de las actividades industriales de fábricas de curtidurías y de alimentos ubicadas en las inmediaciones del río Pachanlica pudieran estar causando un impacto en la calidad del agua en la cuenca. En tal sentido, en el presente estudio se evaluó la calidad del agua de la cuenca del río Pachanlica mediante el uso de índices BMWP (Biological Monitoring Working Party) y EPT (Ephemeroptera, Plecoptera, Trichoptera).

\section{MATERIALES Y MÉTODOS}

\section{Área de estudio}

El estudio fue realizado en la microcuenca del río Pachanlica, la cual es la principal unidad hídrica del río Ambato (figura 1). La microcuenca inicia en las estribaciones de los volcanes Carihuairazo, Chimborazo y nudo del Igualata y presenta una superficie de $376 \mathrm{~km}^{2}$ y $52 \mathrm{~km}$ de longitud. Las entradas anuales de agua son aportadas por la precipitación ( $972 \mathrm{~mm}$ ) y se ubican en alrededor 6,710,2 millones de $\mathrm{m}^{3}$ (Pérez, 2015) 


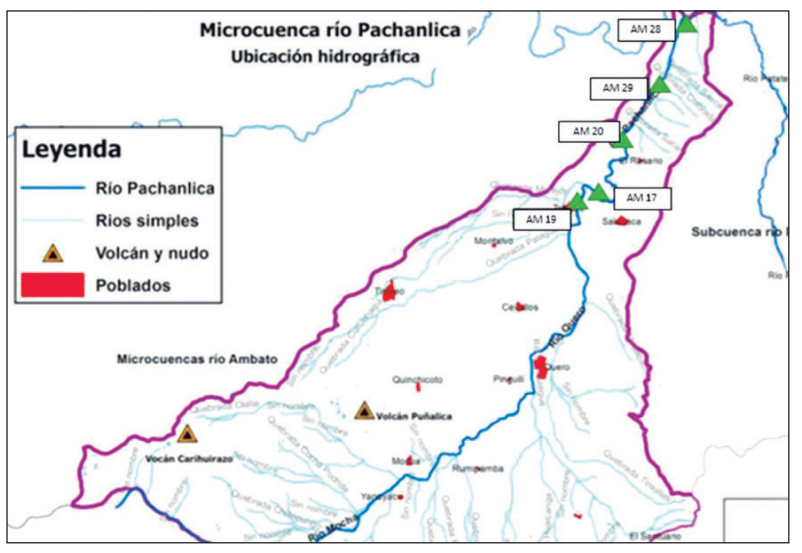

Figura 1. Ubicación de los sitios de muestreo en la microcuenca del río Pachanlica.

La composición faunística y abundancia de macroinvertebrados acuáticos presentes fue determinada mediante muestreos mensuales en cinco puntos (AM19, AM17, AM20, AM29 y AM28) durante dos períodos definidos desde abril-diciembre del 2010 y enero-junio del 2011 (tabla 1). Se recolectó un total de sesenta muestras en ambos períodos.

Tabla 1. Ubicación geográfica de los puntos de muestreo.

\begin{tabular}{|c|c|c|c|c|}
\hline Código & Latitud & Longitud & $\begin{array}{l}\text { Altitud } \\
\text { (m) }\end{array}$ & $\begin{array}{l}\text { Punto de } \\
\text { referencia }\end{array}$ \\
\hline AM 19 & $98^{\circ} 54^{\prime} 21,4^{\prime \prime}$ & $76^{\circ} 79^{\prime} 86^{\prime \prime}$ & 2600 & $\begin{array}{l}\text { Antes de la } \\
\text { descarga }\end{array}$ \\
\hline AM17 & $98^{\circ} 54^{\prime} 23,8^{\prime \prime}$ & $76^{\circ} 80^{\prime} 81^{\prime \prime}$ & 2590 & $\begin{array}{l}\text { Después de } \\
\text { la descarga }\end{array}$ \\
\hline AM 20 & $98^{\circ} 54^{\prime} 26,6^{\prime \prime}$ & $76^{\circ} 79^{\prime} 46^{\prime \prime}$ & 2585 & $\begin{array}{l}\text { Descarga } \\
\text { GELEC }\end{array}$ \\
\hline AM 29 & $98^{\circ} 63^{\prime} 57^{\prime \prime}$ & $77^{\circ} 29^{\prime} 34^{\prime \prime}$ & 2300 & Las Viñas \\
\hline AM 28 & $98^{\circ} 63^{\prime} 70^{\prime \prime}$ & $77^{\circ} 29^{\prime} 10^{\prime \prime}$ & 2290 & $\begin{array}{l}\text { Río } \\
\text { Pachanlica }\end{array}$ \\
\hline
\end{tabular}

El punto AM 19 recibe las aguas residuales de la Curtiduría Artesanal Totoras (Parroquia Totoras, Barrio Palahua). AM 17 recoge descargas del sistema de colectores y alcantarillado del área urbana y rural, así como descargas industriales que en su mayoría no reciben previo tratamiento.

AM 20 recibe las descargas de aguas industriales de la empresa GELEC S.A. (ubicada en Totoras), la cual desde el año 2003 usa ácido sulfúrico en el proceso de acidulación en lugar de ácido sulfuroso (Vasconez
Cabezas, 2005). AM 29: ubicado en una zona rural con predominio de actividades agropecuarias (Choto Costales, 2013). AM 28: ubicado después de la unión de los cauces del río Ambato y Pachanlica, caracterizado por una intensa actividad agrícola.

\section{Captura y recolección de los macroinvertebrados}

La recolecta de los macroinvertebrados en el cauce del río fue hecha usando una red de $250 \mu \mathrm{m}$ colocada a contracorriente (Merritt et al., 2008). Para ello se removieron las piedras y se tomó la muestra conformada por cinco tomas en cada punto de muestreo, siguiendo la metodología de Carrera y Fierro (2001). Los especímenes recolectados fueron colocados en frascos plásticos $(1 \mathrm{~L}$ de volumen) que contenían solución formol-alcohol a 3:1. Cada frasco fue etiquetado y llevado al laboratorio de Biología de la Facultad de Ciencias Agropecuarias de la Universidad Técnica de Ambato (FCAGP-UTA) para su posterior procesamiento e identificación de los especímenes. Una vez en el laboratorio, las muestras se colocaron en bandejas plásticas y observadas bajo aumento de un estereoscopio para la separación de los diferentes morfotipos de macroinvertebrados encontrados. Una vez identificado cada espécimen hasta el nivel Orden y Familia usando la clave taxonómica de Barrios y Puig (2012), se registró el número de individuos/familia para calcular los índices BMWP (Biological Monitoring Working Party) y EPT (Ephemeroptera, Plecoptera, Trichoptera), los cuales han sido usados para la evaluación de calidad del agua en zonas altas de Ecuador (Liñero-Arana et al., 2016) y Argentina (Miserendino et al., 2008).

El índice BMWP es un método sencillo y rápido para evaluar la calidad del agua usando macroinvertebrados mediante el uso de datos son cualitativos (presenciaausencia). Las puntuaciones son determinadas en función de la sensibilidad o tolerancia de diferentes grupos o familias a la contaminación orgánica, en un rango de 1 a 10, con las familias más sensibles designadas con un puntaje de 10, mientras que las más tolerantes a la contaminación son designadas con una puntuación de uno Tabla 2 (Naranjo y López, 2013). Por otra parte, el índice EPT toma en consideración la abundancia de tres órdenes de insectos: Ephemeroptera (mayor tolerancia a la contaminación), Thrichoptera (tolerancia media) y Plecoptera (no tolera la contaminación y solo existe en aguas limpias) (tabla 3 y 4). 
Tabla 2. Valor de tolerancia o susceptibilidad de las diferentes familias de macroinvertebrados para el cálculo del índice BMWP, según Roldán (2003).

Familias

Puntaje

Anamalopsychidae, Atriplectididae, Blepharoceridae, Calamoceratidae, Ptilodactylidae, Chordodidae, Ghomphidae, Hydridae, Lampyridae, Lymessiidae, Odontoceridae, Oliigoneuridae, Perlidae, Polythoridae, Psephenidae

Ampullariidae, Dytiscidae, Ephemeridae, Euthyplociidae, Gyrinidae, Hydraenidae, Hydroboscidae, Leptophlebiidae, Phylopotamidae, Polycentropodidae, Polymitarcyidae, Xiphocentronidae

Gerridae, Hebridae, Helicopsychidae, Hydrobiidae, Leptoceridae, Lestidae, Palaemonidae, Pleidae, Pseudothelpusidae, Saldidae, Simulidae, Veliidae

Baetidae, Caenidae, Calopterygidae, Coenagrionidae, Corixidae, Dixidae, Dryopidae, Glossomatidae, Hyalellidae, Hydropsychidae, Leptohyphidae, Naucoridae, Notonectidae, PLanariidae, Psychodidae, Scirtidae

Aeshnidae, Ancylidae, Corydalidae, Elmidae, Libellulidae, Limnichidae, Lutrochidae, Megapodagrionidae, Sialidae, Staphylinidae

Belostomatidae, Gelastocoridae, Mesoveliidae, Nepidae, Planorbiidae, Pyralidae, Tabanidae, Thiaridae

Chrysomelidae, Stratiomyidae, Haliplidae, Empididae, Dolichopodidae, Sphaeridae, Lymnaeidae, Hydrometridae, Notoceridae

Ceratopogonidae, Glossiphonidae, Cyclobdellidae, Hydrophylidae, Physidae, Tipulidae 3

Culicidae, Chironomidae, Muscidae, Sciomyzidae, Syrphidae 2

Tubificidae

Tabla 3. Valores de referencia del índice BMWP/col. (Roldán, 2003)

\begin{tabular}{lcll}
\hline Calidad & $\begin{array}{c}\text { BMWP/ } \\
\text { Col }\end{array}$ & \multicolumn{1}{c}{ Referencia } & $\begin{array}{c}\text { Color } \\
\text { del agua }\end{array}$ \\
\hline Buena & $>150$, & $\begin{array}{l}\text { Aguas muy limpias } \\
\text { a limpias }\end{array}$ & Azul \\
Aceptable & $61-100$ & $\begin{array}{l}\text { Aguas ligeramente } \\
\text { contaminadas } \\
\text { Aguas }\end{array}$ & Verde \\
Dudosa & $36-60$ & $\begin{array}{l}\text { moderadamente } \\
\text { contaminadas } \\
\text { Aguas muy }\end{array}$ & Amarillo \\
Crítica & $16-35$ & $\begin{array}{l}\text { contaminadas } \\
\text { Aguas fuertemente } \\
\text { contaminadas }\end{array}$ & Rojo \\
\hline $\begin{array}{l}\text { Muy } \\
\text { Crítica }\end{array}$ & $<15$ & & \\
\hline
\end{tabular}

Adicionalmente, en cada punto se tomaron datos físicos-químicos in situ del agua: temperatura, $\mathrm{pH}$, conductividad eléctrica (CE), oxígeno disuelto (OD), salinidad, sólidos totales (SDT), alcalinidad, amonio 44 siguiendo a Franson (1992).
Tabla 4. Valores de referencia para calidad del agua según el índice EPT (Carrera y Fierro 2001)

\begin{tabular}{rrl}
\hline Clase & Índice EPT (\%) & Calidad de agua \\
\hline 1 & $75-100$ & Muy buena \\
2 & $50-75$ & Buena \\
3 & $25-50$ & Regular \\
4 & $0-25$ & Mala \\
\hline
\end{tabular}

\section{RESULTADOS Y DISCUSIÓN}

\section{Caracterización fisicoquímica de la zona de muestreo}

Las variables fisicoquímicas en la microcuenca del río Pachanlica no mostraron diferencias significativas entre las dos épocas de muestreo pero si se observaron variaciones en algunas de estas características entre las diferentes estaciones de muestreo (Tabla 5). La estación AM20 presentó los mayores valores en temperatura $\left(20,78\right.$ y $\left.25,87{ }^{\circ} \mathrm{C}\right)$ y sólidos totales STD 
(2607,80 y 61406,67 mg/L) en la primera y segunda etapa de muestreo, respectivamente y a su vez los menores valores de $\mathrm{pH}(4,81)$ y alcalinidad $(96 \mathrm{ppm})$ durante el primer período de muestreo. Con relación a la conductividad eléctrica, oxígeno disuelto y concentración de amonio no se observaron diferencias significativas entre las estaciones.

\section{Composición faunística}

En general, los macroinvertebrados fueron recolectados fueron encontrados debajo de las piedras y sedimentos. Se encontró un total de 3023 macroinvertebrados, de los cuales 1669 individuos fueron colectados durante el período abril-diciembre 2010 y 1354 individuos durante el segundo período (enero-junio 2011) incluido en ocho órdenes y diez familias (tablas 6 y 7). Durante el primer y segundo período de muestreo, los grupos más abundantes fueron Hyalellidae (Crustacea), seguido de Tubificidae (Annelida) y Chironomidae (Diptera). Contrariamente, los grupos menos comunes fueron Baetidae (Ephemeroptera), Leptoceridae (Trichoptera) y Elmidae (Coleoptera) durante el primer período de muestreo, mientras que durante el segundo período los menos abundantes fueron Ceratopogonidae (Diptera) y Macrobdellidae (Hirudinea). Así mismo, Medina-Tafur et al. (2010) registraron la presencia de siete Clases, trece Órdenes y 46 Familias, constituidos por las clases Insecta, Gastropoda, Crustacea, Bivalvia, Turberllaria, Oligoqueta y Ostracoda en suelos de calidad variable en el Alto Chicama, Perú. Sin embargo, esta composición fue variable a lo largo del sitio de muestreo. Según Oyague-Passuni y Maldonado-Fonkén (2015), esta variabilidad en la composición de macroinvertebrados acuáticos está relacionada con la heterogeneidad espacial de los sitios de muestreo que es influenciada por la estructura física, la calidad del agua y las comunidades de plantas.

Tabla 5. Variables físicas y químicas evaluadas durante dos etapas de muestreo en la microcuenca del río Pachanlica

\begin{tabular}{|c|c|c|c|c|c|c|c|c|}
\hline & $\mathrm{T}\left({ }^{\circ} \mathrm{C}\right)$ & $\mathrm{pH}$ & $\begin{array}{l}\text { Conductividad } \\
\qquad(\mu \mathrm{S} / \mathrm{cm})\end{array}$ & $\begin{array}{c}\text { Oxígeno } \\
\text { disuelto (\%) }\end{array}$ & Salinidad & $\begin{array}{c}\text { Sólidos totales } \\
(\mathrm{mg} / \mathrm{L})\end{array}$ & $\begin{array}{l}\text { Alcalinidad } \\
\text { (ppm) }\end{array}$ & $\begin{array}{l}\text { Amonio } \\
\text { (ppm) }\end{array}$ \\
\hline \multicolumn{9}{|c|}{ abril-diciembre del 2010} \\
\hline AM17 & $16,40 \pm 1,46 b$ & $8,34 \pm 0,48 a$ & $500,48 \pm 278,77 a$ & $57,24 \pm 25,36 a$ & $376,50 \pm 129,14 a$ & $461,17 \pm 95,82 b$ & $180 \pm 0,00 \mathrm{a}$ & $0,71 \pm 0,26 a$ \\
\hline AM19 & $17,10 \pm 2,52 \mathrm{ab}$ & $8,33 \pm 0,45 a$ & $534,20 \pm 63,63 a$ & $66,08 \pm 3,66 a$ & $319,50 \pm 37,11 a$ & $884,33 \pm 1160,14 b$ & $180 \pm 0,00 \mathrm{a}$ & $3,79 \pm 7,17 a$ \\
\hline AM20 & $20,78 \pm 2,61 a$ & $4,81 \pm 1,51 b$ & $324,25 \pm 106,16 a$ & $67,23 \pm 0,91 a$ & $132,16 \pm 60,01 a$ & $2607,80 \pm 731,15 a$ & $96,00 \pm 70,00 \mathrm{~b}$ & $0,83 \pm 0,75 a$ \\
\hline AM28 & $17,76 \pm 1,85 \mathrm{ab}$ & $8,46 \pm 0,26 a$ & $532,20 \pm 283,09 a$ & $76,42 \pm 13,70 a$ & $296,86 \pm 130,63 a$ & $412,71 \pm 2539,08 b$ & $180 \pm 0,00 \mathrm{a}$ & $0,89 \pm 0,56 a$ \\
\hline AM29 & $17,66 \pm 1,95 \mathrm{ab}$ & $8,37 \pm 0,20 a$ & $587,80 \pm 229,51 a$ & $76,48 \pm 13,58 a$ & $288,00 \pm 88,68 a$ & $1561,90 \pm 2493,25 a b$ & $175,71 \pm 0,00 a$ & $1,00 \pm 0,63 a$ \\
\hline \multicolumn{9}{|c|}{ enero-junio del 2011} \\
\hline AM17 & $16,96 \pm 0,72 b$ & $8,18 \pm 0,157 a$ & $530,40 \pm 190,02 a$ & $67,65 \pm 60,12 a$ & $261,00 \pm 93,11 a$ & $404,40 \pm 108,40 b$ & $148,00 \pm 43,81 a$ & $0,25 \pm 0,11 a$ \\
\hline AM19 & $15,94 \pm 0,98 b$ & $8,23 \pm 0,301 a$ & $405,80 \pm 153,99 a$ & $262,58 \pm 392,28 a$ & $247,80 \pm 45,43 a$ & $311,00 \pm 79,61 b$ & $148,00 \pm 43,81 a$ & $0,50 \pm 0,25 a$ \\
\hline AM20 & $25,87 \pm 3,10 a$ & $8,42 \pm 3,007 a$ & $334,33 \pm 88,54 a$ & $109,27 \pm 29,97 a$ & $160,33 \pm 46,06 a$ & $61406,67 \pm 10183,56 a$ & $140,00 \pm 90,18 a$ & $1,50 \pm 0,86 a$ \\
\hline AM28 & $15,88 \pm 1,10 b$ & $8,58 \pm 0,162 a$ & $495,60 \pm 161,30 a$ & $66,46 \pm 54,15 a$ & $279,00 \pm 47,15 a$ & $407,60 \pm 60,39 b$ & $160,00 \pm 34,64 a$ & $0,75 \pm 0,44 a$ \\
\hline AM29 & $16,06 \pm 2,27 b$ & $8,18 \pm 0,193 a$ & $381,36 \pm 225,45 a$ & $84,38 \pm 50,53 a$ & $196,52 \pm 116,37 a$ & $304,25 \pm 146,38 b$ & $148,00 \pm 43,81 a$ & $1,00 \pm 0,44 a$ \\
\hline
\end{tabular}

Basados en el índice BMWP mostrados en la tabla 8, en general, las zonas de muestreo pueden ser categorizadas como aguas de calidad muy crítica (punto AM20 donde se encuentra la descarga de GELEC) hasta calidad crítica en el resto de los puntos muestreados puesto que se observaron valores entre 22 y 34 . De manera similar, durante la segunda fecha de muestreo, la mayoría de los puntos mostraron valores de BMWP entre 23 y 36 , excepto el punto AM20.

Así mismo, los valores del índice EPT estuvieron por debajo del $25 \%$, lo que permite confirmar que las aguas de la cuenca del río Pachanlica pueden ser categorizadas como de mala calidad de acuerdo a la escala de Carrera 
y Fierro (2001). Esta situación fue aún más evidente en los puntos AM20 y AM28 durante el muestreo desde abril a diciembre del 2010 y en los puntos AM17 y AM20 durante el segundo período de muestreo (enero-junio del 2011). Durante la segunda etapa se observó un ligero incremento en los puntos AM17, AM19 y AM29, aún cuando estos valores siguen estando en niveles críticos de contaminación.

Con base a el índice BMWP, Medina-Tafur et al. (2010) se encontró que las microcuencas Perejil, Caballo Moro y Chuyugual mostraron calidad diferencial dependiendo de la ubicación, la cual varió desde aguas con calidad biológica regular, aguas con calidad biológica aceptable en áreas sin mucha influencia del hombre hasta aguas de mala calidad en las zonas más bajas de la cuenca. Estos autores concluyen que las condiciones físicoquímicas están siendo alteradas en estos ríos debido a las actividades de minería, ganadería y agricultura, así como a la mala disposición de las aguas residuales de los centros poblados. Sánchez-Herrera (2005) encontró que las aguas del río Pamplonita, en Colombia presentaban diferentes clases de calidad que variaron desde la clase II (aguas con evidencias de contaminación) hasta clase $\mathrm{V}$ (aguas fuertemente contaminadas).
Aunque el índice biológico BMWP ha mostrado ser bastante útil en la evaluación de la calidad del agua, los valores que han sido asignados a las diferentes clases de calidad deberían ser ajustados considerando las condiciones geológicas, de pendiente, de altura y de sustrato de las corrientes en cada región, de manera de hacer que las ponderaciones de sensibilidad a los rangos de tolerancia ambiental de los macroinvertebrados acuáticos sean más exactas (Roldán, 2003; Sánchez-Herrera, 2005).

Con relación al índice EPT, en Malasia Ab Hamid y Md Rawi (2014), observaron composición similar de los diferentes taxones de Ephemeroptera, Plecoptera y Trichoptera en los ríos Tupah, Batu Hampar, pero observaron menos taxones comunes con el río Teroi, aunque en general el índice de riqueza de taxones del EPT indicó que la calidad del agua en los tres ríos no fue afectada por las actividades de los visitantes. Entre las limitantes de este tipo de índice, es que su capacidad de explicación de la contaminación del agua está limitada cuando se usa en áreas donde de manera natural o debido a la acción del ser humano muestre baja riqueza de especies de estos grupos de insectos 0 en áreas donde exista interés en otros grupos tolerantes a la contaminación.

Tabla 6. Abundancia (\# de individuos) de macroinvertebrados recolectados en la microcuenca del río Pachanlica (período abril-diciembre del 2010).

\begin{tabular}{lllrrrrrr}
\hline \multirow{2}{*}{ Phylum/Subphylum } & $\begin{array}{c}\text { Orden } \\
\text { o Subclase }\end{array}$ & Familia & \multicolumn{1}{c}{} \\
\cline { 3 - 8 } & & AM17 & AM19 & AM20 & AM28 & AM29 & Total \\
\hline Arthropoda/Insecta & Ephemeroptera & Baetidae & 0 & 0 & 0 & 2 & 0 & 2 \\
& Trichoptera & Hidrobiosidae & 0 & 25 & 0 & 0 & 0 & 25 \\
& Diptera & Leptoceridae & 0 & 0 & 0 & 0 & 8 & 8 \\
& & Chironomidae & 65 & 315 & 9 & 18 & 24 & 431 \\
& Coleoptera & Elmidae & 2 & 2 & 0 & 0 & 0 & 4 \\
& Neuroptera & Corydalidae & 8 & 0 & 0 & 0 & 20 & 28 \\
Arthropoda/Crustacea & Amphipoda & Hyalellidae & 314 & 1 & 0 & 286 & 23 & 624 \\
Annelida & Clitellata & Tubificidae & 137 & 254 & 0 & 44 & 39 & 474 \\
& Hirudinea & Glossiphonidae & 3 & 0 & 0 & 4 & 52 & 59 \\
Total & & 535 & 602 & 9 & 357 & 166 & 1669 \\
\hline
\end{tabular}


Probablemente, la relativa poca abundancia de estos grupos de insectos en la zona de estudio podría tener relación con los parámetros físico-químicos del agua. En este sentido, la concentración de nitrógeno influye en la abundancia y diversidad de macroinvertebrados acuáticos (Begon et al., 2006). En el presente estudio, la baja abundancia de macroinvertebrados podría ser explicada por los altos niveles de $\mathrm{NH}_{4}^{+}$, los cuales excedían los límites máximos tolerados por la mayoría de los organismos acuáticos $(0,1 \mathrm{mg} / \mathrm{L})$ (Santhosh y Singh, 2007). Adicionalmente, el incremento del amonio, junto con la disminución de oxígeno disuelto en el agua reduce el valor del índice BMWQ (Alonso et al., 2002). Por otra parte, Molina et al. (2008) observaron que otros factores como la conductividad eléctrica, los sólidos totales, el pH y el oxígeno disuelto inciden de manera diferencial con la abundancia dependiendo del grupo de macroinvertebrados considerado.

En general, la baja abundancia de organismos indicadores de buena calidad del agua obtenida en el río Pachanlica indica la existencia de un índice de contaminación crítico, el cual muestra en algunos de los puntos de muestreo una clara tendencia a incrementar. De acuerdo con García y Rosas (2010), la ausencia de estos grupos de macroinvertebrados que sirven como indicadores de contaminación evidencian cambios ecológicos negativos en las zonas de muestreo, relacionados con el impacto de la actividad antrópica. En los diferentes puntos de monitoreo se observa actividad de la industria alimenticia (elaboración de gelatinas), embotelladoras, curtiembres, agrícola (cultivos de ciclo corto) y una alta incidencia de aguas domésticas. En tal sentido, las intensas actividades antrópicas (comerciales, industriales, agricultura, aguas servidas) que tienen lugar en la zona de estudio podrían haber tenido influencia en los altos niveles de contaminación observados en algunos puntos de monitoreo.

\section{CONCLUSIONES}

Los valores tanto del índice BMWP/col como EPT en los diferentes puntos monitoreados en la microcuenca del río Pachanlica durante la primera y segunda fechas de muestreo mostraron que existen niveles de contaminación del agua que pudieran ser considerados alarmantes. En tal sentido, deberían ser tomadas medidas de saneamiento que paulatinamente disminuyan la contaminación de las aguas.

\section{AGRADECIMIENTOS}

Los autores agradecen a la Universidad Técnica de Ambato principalmente a la Facultad de Ciencias Agropecuarias, a la Secretaría Nacional del Agua (SENAGUA) por el apoyo técnico, científico y logístico prestado a la presente investigación.

\section{REFERENCIAS}

Ab Hamid, S. y Md Rawi, C.S. 2014. Ecology of Ephemeroptera, Plecoptera and Trichoptera (Insecta) in Rivers of the Gunung Jerai Forest Reserve: Diversity and Distribution of Functional Feeding Groups. Tropical Life Sciences Research 25(1): 61-73.

Armenteras, D., Gast, F. y Villareal, H. 2003. Andean forest fragmentation and the representativeness of protected natural areas in the eastern Andes, Colombia. Biological Conservation 113: 245-256.

Barrios, E. y Puig A. 2012. Catálogo y claves de identificación de organismos invertebrados utilizados como elementos de calidad en las redes de control del estado ecológico. Ministerio de Agricultura, Alimentación y Medio Ambiente, Madrid, España.

Basset A., Sangiorgio, F. y Pinna, M. 2004. Monitoring with benthic macroinvertebrates: advantages and disadvantages of body size descriptors. Aquatic Conservation: Marine and Freshwater Ecosystems 14: 43-58.

Buytaert, W., Célleri, R., De Bièvre, B., Cisneros F., Wyseure, G., Deckers J. y Hofstede R. 2006. Human impact on the hydrology of the Andean paramos. Earth-Science Reviews 79: 53-72.

Carrera, C. y Fierro, C. 2001. Manual de Monitoreo. Los macroinvertebrados acuáticos como indicadores de la calidad del agua. Ecociencia, Quito.

Cummnig, K. y Klug, J. 1979. Feeding ecology of stream invertebrates. Annual Review of Ecology, Evolution, and Systematics 10: 147-172.

García, E. y Rosas, K. 2010. Biodiversidad de insectos acuáticos asociados a la Cuenca del Río Grande Manatí. Departamento de Recursos Naturales y Ambientales (DRNA), Puerto Rico http://drna.pr.gov/historico/ oficinas/saux/secretaria-auxiliar-de-planificacion tegral/ planagua/proyectos-degeointernado/Biodiversidad_2010_ FINAL.pdf/vie. Consultado: 18 julio de 2017. 
Franson M.A. 1992. Métodos normalizados para el análisis de aguas potables y residuales. Ediciones Díaz de Santos, S.A. Madrid, España.

Guimarães, M.E.E., Mascarenhas, A., Sousa, C., Boski, T., Dentinho, T.P. 2012. The impact of water quality changes on the socio-economic system of the Guadiana Estuary: an assessment of management options. Ecology and Society 17(3): 38-51.

Gutiérrez-Yurrita, P.J., Morales-Ortiz, A., Oviedo, A. y Ramírez, C. 2002. Distribution, spread, habitat characterization and conservation of the crayfish species (Cambaridae) in Querétaro (Central México). Freshwater Crayfish 13: 288-297.

Jara, C. 2002. Evaluación de la existencia de insectos bioindicadores de la calidad del agua en zonas retrónicas y potámicas de tres ríos de la zona semiárida de Chile. Tesis de Grado, Universidad de Chile, Santiago, Chile.

Jorcin, A. y Nogueira, M.G. 2008. Benthic macroinvertebrates in the Paranapanema reservoir cascade (southeast Brazil). Brazilian Journal of Biology 68(4): 1013-1024.

Liñero-Arana I., Balarezzo, V.H., Eraso, H., Pacheco, F., Ramos, C.E., Muzo, R.G. y Calva, C.J. 2016. Calidad del agua de un río andino ecuatoriano a través del uso de macroinvertebrados. Cuadernos de Investigación UNED 8(1): 68-75.

Medina-Tafur, C.M., Hora-Revilla, I., Ascencio-Gusmán, Pereda-Ruíz, W. y Gabriel-Aguilar, R. 2010. Índice Biological Monitoring Working Party (BMWP), modificado y adaptado a tres microcuencas del Alto Chicana. La Libertad, Perú. Uniciencia 1:5-20.

Merritt, R.W., Cummins, K.W. y Berg, M.B. 2008. An introduction to the aquatic insects of North America. Hunt Publishing Company, Kendall, USA.

Miserendino, M.L., Brand, C. y Di Prinzio, C. 2008. Assessing urban impacts on water quality, benthic communities and fish in streams of the Andes mountains, Patagonia (Argentina). Water Air and Soil Pollution 194(1):91-110.
Molina, C., François-Marie G., Pinto, J. y Rosales, C. 2008. Estructura de macroinvertebrados acuáticos en un río altoandino de la Cordillera Real, Bolivia: variación anual y longitudinal en relación a factores ambientales. Ecología Aplicada 7(1-2): 105-116.

Monar, C., Saavedra, A.K., Escudero, L., Delgado, J.A., Alwang, J., Barrera, V. y Botello, R. 2013. Positive impacts in soil and water conservation in an Andean region of South America: Case scenarios from a US Agency for International Development multidisciplinary cooperative project. Journal of Soil and Water Conservation 68(1):25-30.

Montoya, Y. y Aguirre, N. 2009. Estado del arte de la limnología de lagos de planos inundables (Ciénagas) en Colombia. Revista Gestión y Ambiente 12(3): 85-106.

Naranjo, J., y López, P. 2013. Biological monitoring working party, un índice biótico con potencialidades para evaluar la calidad de las aguas en ríos cubanos. Ciencia en su PC 2: 15-25.

Norris, R.H. y Hawkins, C.P. 2000. Monitoring river health. Hydrobiologia 435: 5-17.

Oyague-Passuni, E. y Maldonado-Fonkén, M.S. 2015. Relationships between aquatic invertebrates, water quality and vegetation in an Andean peatland system. Mires and Peat 15: 1-21.

Pérez, S. 2015. Gestión Actual de los Recursos Hídricos en la Subcuenca del río Ambato desde los Actores. Gobierno Provincial de Tungurahua, Ecuador.http://rrnn.tungurahua. gob.ec/documentos/ver/56cc9a4283ba88c90ac8c289. Consultado: 27 junio 2017.

Reinoso, G., Gutiérrez, C., López, E., Carranza, X. y Vásquez, J. 2008. Macroinvertebrados acuáticos. En: Reinoso, G., Navarro, F., Melo, J., Vejarano, M. y Esquivel, H. Editores. Biodiversidad faunística y florística de la subcuenca del río Anamichú, (Cuenca del río Saldaña), Biodiversidad Regional Fase IV. Universidad del Tolima, Ibagué, Colombia.

Rivera, J., Camacho, D. y Votero, A. 2008. Estructura numérica de la entomofauna acuática en ocho quebradas del Departamento del Quindío-Colombia. Acta Biológica Colombiana 13(2): 133-146. 
Roldán, G. 2003. La bioindicación de la calidad del agua en Colombia, propuesta para el uso del método BMWP-COL. Colección ciencia y tecnología. Editorial Universidad de Antioquia, Medellín.

Rosenberg, D.M. y Resh, V.H. 1993. Freshwater biomonitoring and benthic macroinvertebrates. Chapman \& Hall, New York.

Sánchez-Herrera, M.J. 2005. El índice biológico BMWP (Biological Monitoring Working Party score), modificado y adaptado al cauce principal del río Pamplonita Norte de Santander. Bistua: Revista de la Facultad de Ciencias Básicas 3(2): 54-67.

Seaonez, C. 1999. Aguas urbanas, tratamientos naturales de bajo costo y aprovechamiento. Mundiprensa, España.

Vázquez-Silva, G., Castro-Mejía, G., González-Mora, I., Pérez Rodríguez, R. y Castro-Barrera, T. 2006. Bioindicadores como herramientas para determinar la calidad del agua. Contactos 60: 41-48.

Fecha de recepción: 15/01/2018

Fecha de aceptación: 26/05/2018

Publicado en línea: 28/05/2018

Para citar este artículo: Buenaño, M., Zurita-Vásquez, H., Parra, G. y Pérez, R. 2018. Macroinvertebrados bentónicos como indicadores de calidad de agua en la cuenca del Pachanlica, provincia de Tungurahua, Ecuador. Intropica 13(1): 41-49.

DOI: http://dx.doi.org/10.21676/23897864.2405 